\title{
CONCERNING PERIODIC POINTS IN MAPPINGS OF CONTINUA
}

\author{
W. T. INGRAM
}

(Communicated by Dennis Burke)

\begin{abstract}
In this paper we present some conditions which are sufficient for a mapping to have periodic points.

THEOREM. If $f$ is a mapping of the space $X$ into $X$ and there exist subcontinua $H$ and $K$ of $X$ such that (1) every subcontinuum of $K$ has the fixed point property, (2) $f[K]$ and every subcontinuum of $f[H]$ are in class $W,(3) f[K]$ contains $H$, (4) $f[H]$ contains $H \cup K$, and (5) if $n$ is a positive integer such that $(f \mid H)^{-n}(K)$ intersects $K$, then $n=2$, then $K$ contains periodic points of $f$ of every period greater than 1.

Also included is a fixed point lemma:

LEMMA. Suppose $f$ is a mapping of the space $X$ into $X$ and $K$ is a subcontinuum of $X$ such that $f[K]$ contains $K$. If (1) every subcontinuum of $K$ has the fixed point property, and (2) every subcontinuum of $f[K]$ is in class $W$, then there is a point $x$ of $K$ such that $f(x)=x$.

Further we show that: If $f$ is a mapping of $[0,1]$ into $[0,1]$ and $f$ has a periodic point which is not a power of 2 , then $\lim \{[0,1], f\}$ contains an indecomposable continuum. Moreover, for each positive integer $i$, there is a mapping of $[0,1]$ into $[0,1]$ with a periodic point of period $2^{i}$ and having a hereditarily decomposable inverse limit.
\end{abstract}

1. Introduction. In his book, An Introduction to Chaotic Dynamical Systems [3, Theorem 10.2, p. 62], Robert L. Devaney includes a proof of Sarkovskii s' Theorem. Consider the following order on the natural numbers: $3 \triangleright 5 \triangleright 7 \triangleright \cdots \triangleright 2 \cdot 3 \triangleright$ $2 \cdot 5 \triangleright \cdots \triangleright 2^{2} \cdot 3 \triangleright 2^{2} \cdot 5 \triangleright \cdots \triangleright 2^{3} \cdot 3 \triangleright 2^{3} \cdot 5 \triangleright \cdots \triangleright 2^{3} \triangleright 2^{2} \triangleright 2 \triangleright 1$. Suppose $f: R \rightarrow R$ is continuous. If $k \triangleright m$ and $f$ has a periodic point of prime period $k$, then $f$ has a periodic point of period $m$. In working through a proof of this theorem for $k=3$, the author discovered the main result of this paper-Theorem 2. For an alternate proof of Sarkovskii's Theorem for $k=3$, see also [7]. For a further look at this theorem for ordered spaces see [13].

By a continuum we mean a compact connected metric space and by a mapping we mean a continuous function. By a periodic point of period $n$ for a mapping $f$ of a continuum $M$ into $M$ is meant a point $x$ such that $f^{n}(x)=x$. The statement that $x$ has prime period $n$ means that $n$ is the least integer $k$ such that $f^{k}(x)=x$. A continuum $M$ is said to have the fixed point property provided if $f$ is a mapping of $M$ into $M$ there is a point $x$ such that $f(x)=x$. A mapping $f$ of a continuum $X$ onto a continuum $M$ is said to be weakly confluent provided for each subcontinuum $K$ of

Received by the editors May 22, 1987 and, in revised form, September 14, 1987.

1980 Mathematics Subject (lassification (1985 Revision). Primary 54F20, 54H20; Secondary 54F62, 54H25, 54F55.

Key words and phrases. Periodic point, fixed point property, class $W$, indecomposable continuum, inverse limit. 
$M$ some component of $f^{-1}(K)$ is thrown by $f$ onto $K$. A continuum is said to be in Class $W$ provided every mapping of a continuum onto it is weakly confluent. The continuum $T$ is a triod provided there is a subcontinuum $K$ of $T$ such that $T-K$ has at least three components. A continuum is atriodic provided it does not contain a triod. A continuum $M$ is unicoherent provided if $M$ is the union of two subcontinua $H$ and $K$, then the common part of $H$ and $K$ is connected. A continuum is hereditarily unicoherent provided each of its subcontinua is unicoherent. If $f$ is a mapping of a space $X$ into $X$, the inverse limit of the inverse limit sequence $\left\{X_{i}, f_{i}\right\}$ where, for each $i, X_{i}$ is $X$ and $f_{i}$ is $f$ will be denoted $\lim \{X, f\}$. For the inverse sequence $\left\{X_{i}, f_{i}\right\}$, the inverse limit is the subset of the product of the sequence of spaces $X_{1}, X_{2}, \ldots$ to which the point $\left(x_{1}, x_{2}, \ldots\right)$ belongs if and only if $f_{i}\left(x_{i+1}\right)=x_{i}$.

There has been considerable interest in periodic homeomorphisms of continua where a homeomorphism $h$ is called periodic provided there is an integer $n$ such that $h^{n}$ is the identity. Wayne Lewis has shown [8] that for each $n$ there is a chainable continuum with a periodic homeomorphism of period $n$. A theorem of Michel Smith and Sam Young [14] should be compared with Theorem 3 of this paper. Smith and Young show that if a chainable continuum $M$ has a periodic homeomorphism of period greater than 2, then $M$ contains an indecomposable continuum. In this paper we consider the question of the existence of periodic points in mappings of continua.

2. A fixed point theorem. The problem of finding a periodic point of period $n$ for a mapping $f$ is, of course, the same as the problem of finding a fixed point for $f^{n}$. Not surprisingly, we need a fixed point theorem as a lemma to the main theorem of this paper. The following theorem, which the author finds interesting in its own right, should be compared with an example of Sam Nadler [11] of a mapping with no fixed point of a disk to a containing disk. A corollary to Theorem 1 is the well-known corresponding result for mappings of intervals.

THEOREM 1. Suppose $X$ is a space, $f$ is a mapping of $X$ into $X$, and $K$ is a subcontinuum of $X$ such that $f[K]$ contains $K$. If (1) every subcontinuum of $K$ has the fixed point property, and (2) every subcontinuum of $f[K]$ is in Class $W$, then there is a point $x$ of $K$ such that $f(x)=x$.

Proof. Since $f[K]$ is in Class $W$ and $K$ is a subset of $f[K]$, there is a subcontinuum $K_{1}$ of $K$ such that $f\left[K_{1}\right]=K$. Then $f \mid K_{1}: K_{1} \rightarrow K$ is weakly confluent since every subcontinuum of $f[K]$ is in Class $W$; thus there is a subcontinuum $K_{2}$ of $K_{1}$ such that $f\left[K_{2}\right]=K_{1}$. Since $K_{1}$ is in Class $W, f \mid K_{2}: K_{2} \rightarrow K_{1}$ is weakly confluent; therefore there is a subcontinuum $K_{3}$ of $K_{2}$ such that $f\left[K_{3}\right]=K_{2}$. Continuing this process there exists a monotonic decreasing sequence $K_{1}, K_{2}, K_{3}, \ldots$ of subcontinua of $K$ such that $f\left[K_{i+1}\right]=K_{i}$ for $i=1,2,3, \ldots$. Let $H$ denote the common part of all the terms of this sequence and note that $f[H]=H$, since $f[H]=f\left[\bigcap_{i>0} K_{i}\right]=\bigcap_{i>0} f\left[K_{i}\right]=\bigcap_{i>0} K_{i}=H$. Since $f \mid H$ throws $H$ onto $H$ and $H$ has the fixed point property, there exists a point $x$ of $H$ (and therefore of $K$ ) such that $f(x)=x$.

REMARK. Note that (1) and (2) of the hypothesis of Theorem 1 are met if $f[K]$ is chainable ([12, Theorem 4, p. 236 and 4], respectively), while (2) is met if $f[K]$ is 
atriodic and acyclic [1] and (1) is met by planar, tree-like continua such that each two points of a subcontinuum $L$ lie in a weakly chainable subcontinuum of $L[\mathbf{1 0}]$.

3. Periodic points. In this section we prove the main result of the paper.

THEOREM 2. If $f$ is a mapping of the space $X$ into $X$ and there exist subcontinua $H$ and $K$ of $X$ such that (1) every subcontinuum of $K$ has the fixed point property, (2) $f[K]$ and every subcontinuum of $f[H]$ are in class $W,(3) f[K]$ contains $H$, (4) $f[H]$ contains $H \cup K$, and (5) if $n$ is a positive integer such that $(f \mid H)^{-n}(K)$ intersects $K$, then $n=2$, then $K$ contains periodic points of $f$ of every period greater than 1 .

ProOF. Suppose $n \geq 2$. There is a sequence $H_{1}, H_{2}, \ldots, H_{n-1}$ of subcontinua of $H$ such that $f\left[H_{1}\right]=K$ (note that $f \mid H$ is weakly confluent) and $f\left[H_{i+1}\right]=H_{i}$ for $i=1,2, \ldots, n-2$ (in case $n>2$ ). There is a subcontinuum $K_{n}$ of $K$ so that $f\left[K_{n}\right]=H_{n-1}$. Thus, $f^{n}\left[K_{n}\right]=K$ and so $f^{n}\left[K_{n}\right]$ contains $K_{n}$, so, by Theorem 1 , there is a point $x$ of $K_{n}$ such that $f^{n}(x)=x$. We must show that if $j<n$ then $f^{j}(x)$ is not $x$. If $j<n$ and $f^{j}(x)=x$, then $j=n-2$ and $x$ is in $H_{2}$. Since $f^{n}(x)=x$ and $f^{n-2}(x)=x, f^{2}(x)=x$. Since $x$ is in $(f \mid H)^{-2}(K), x$ is in $(f \mid H)^{-4}(K)$ and in $K$ contrary to (5) of the hypothesis. Therefore, $x$ is periodic of prime period $n$.

REMARK. If $f$ is a mapping of the continuum $M$ into itself and $f$ has a periodic point of period $k$, then the mapping of $\lim \{M, f\}$ induced by $f$ has periodic points of period $k$, e.g. $\left(x, f^{k-1}(x), \ldots, f(x), x, \ldots\right)$. Thus, although Theorem 2 does not directly apply to homeomorphisms, it may be used to conclude the existence of homeomorphisms with periodic points.

COROLlARY. If $M$ is a chainable continuum, $f$ is a mapping of $M$ into $M$, and there are subcontinua $H$ and $K$ of $M$ such that $f[K]=H, f[H]$ contains $H \cup K$, and if $(f \mid H)^{-n}(K)$ intersects $K$ then $n=2$ then $f$ has periodic points of every period.

B

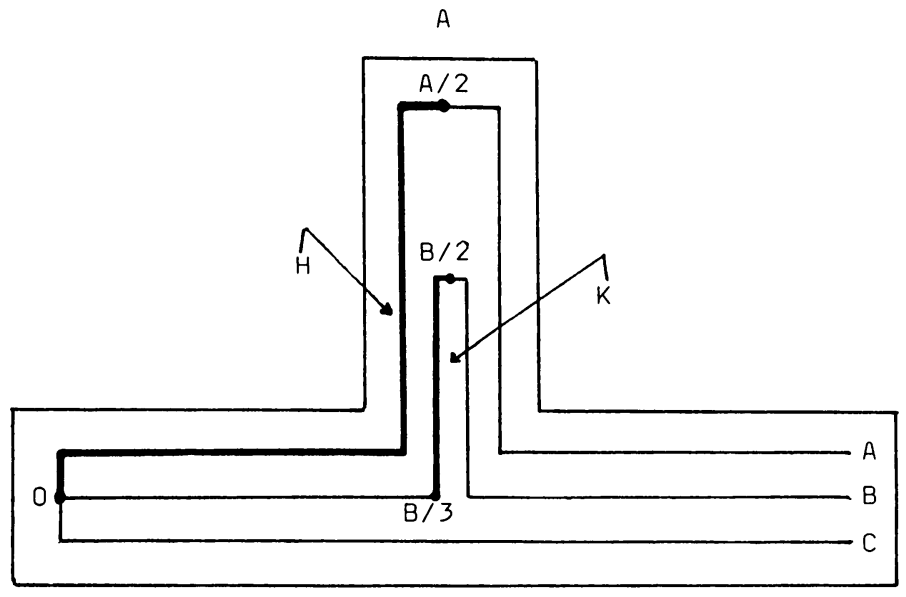


EXAMPLE. Let $f$ be the mapping of the simple triod $T$ to itself given in [5]. The mapping $f$ is represented in Figure 1 above. Letting $H=[0, A / 2]$ and $K=$ $[B / 3, B / 2]$ it follows from Theorem 2 that $f$ has periodic points of every period.

EXAMPLE. Let $f$ be the mapping of the simple triod $T$ to itself given in [2]. The mapping $f$ is represented in Figure 2 below. Letting $H=[0,3 B / 8]$ and $K=[C / 32, C / 8]$, it follows from Theorem 2 that $f$ has periodic points of every period.

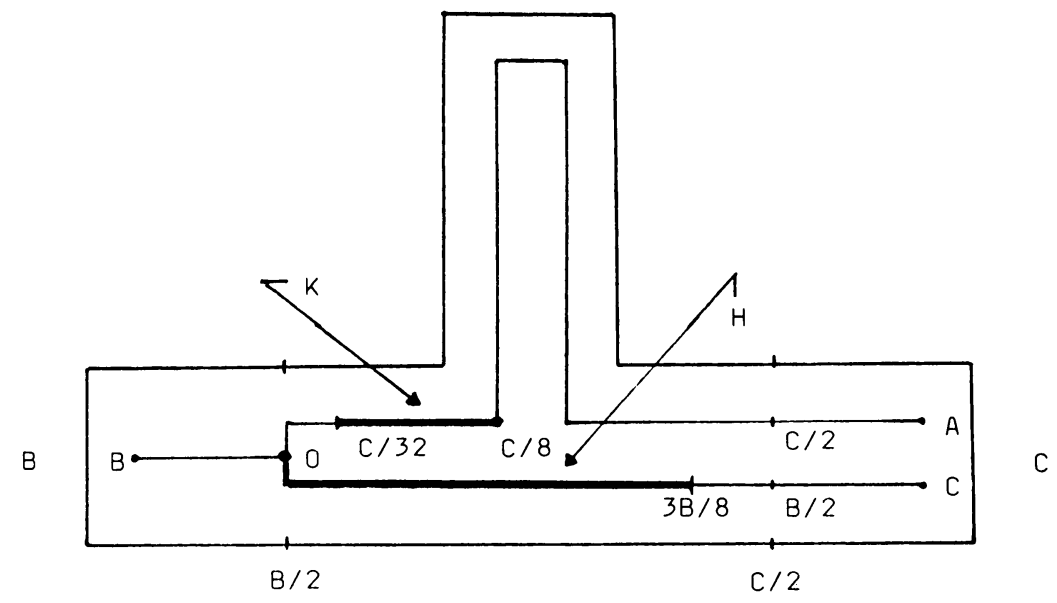

FIGURE 2

EXAMPLE. Let $f$ be the mapping of the unit circle $S^{1}$ to itself given by $f(z)=z^{2}$. Letting $H=\left\{e^{i \theta} \mid 0 \leq \theta \leq 3 \pi / 4\right\}$ and $K=\left\{e^{i \theta} \mid \pi \leq \theta \leq 3 \pi / 2\right\}$, it follows from Theorem 2 that $f$ has periodic points of every period. Similarly, if $f$ is a mapping of $S^{1}$ onto itself which is homotopic to $z^{n}$ for some $n>1$, then $f$ has periodic points of every period.
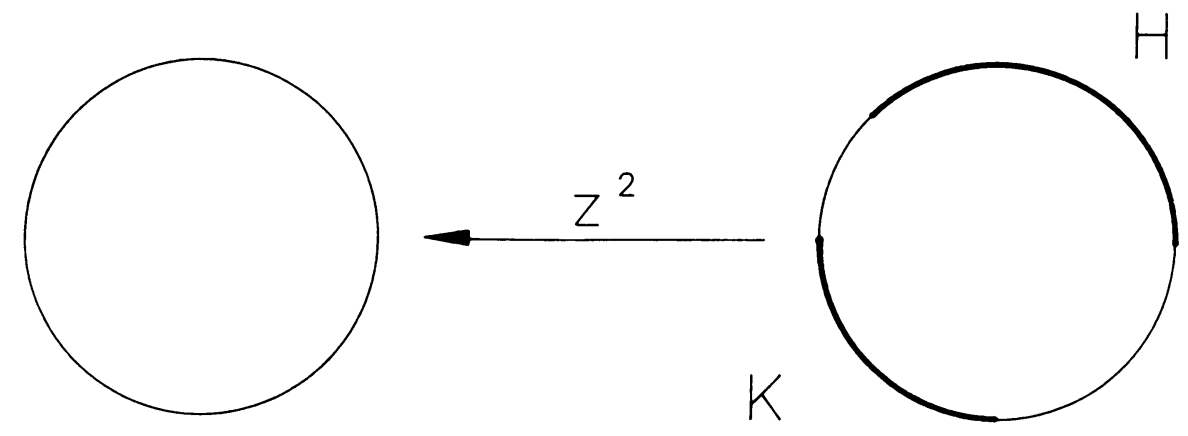

FIGURE 3

COROLLARY. If $f$ is a mapping of an interval to itself with a periodic point of period 3 , then $f$ has periodic points of every period. 
PROOF. To see this it is a matter of noting that the hypothesis of Theorem 2 is met. We indicate the proof for one of two cases and leave the second similar case to the reader. Suppose $a, b$ and $c$ are points of the interval with $a<b<c$ and $f(a)=b, f(b)=c$ and $f(c)=a$ [the other case is $f(a)=c, f(b)=a$ and $f(c)=b]$.

If $f^{-1}(c)$ is nondegenerate, then there exist mutually exclusive intervals $H$ and $K$ lying in $[b, c]$ and $[a, b]$, respectively, so that $f[H]$ is $[a, c]$ and $f[K]$ is $[b, c]$ and Theorem 2 applies.

Suppose $f^{-1}(c)=\{b\}$. Choose $K$ lying in $[a, b]$ and $H$ lying in $[b, c]$ so that $f[K]=[b, c]$ and $f[H]=[a, c]$. For each $i$, denote by $H_{i}$ the set $(f \mid H)^{-1}(K)$. Note that $a$ is not in $H_{i}$ for $i=1,2,3, \ldots$ so $c$ is not in $H_{i}$ for $i=2,3,4, \ldots$ and thus $b$ is not in $H_{i}$ for $i=3,4, \ldots$ Further, $b$ is not in $H_{1}$ since $c$ is not in $K$. Thus, if $H_{i}$ intersects $K$, then $i=2$. Consequently, the hypothesis of Theorem 2 is met.

REMARK. Condition (5) of Theorem 2 seems a bit artificial. A more natural condition the author experimented with in its place is a requirement that $H$ and $K$ be mutually exclusive. In fact, in each of the examples, the $H$ and $K$ given are mutually exclusive. However, replacing condition (5) with this proved to be undesirable in that the Sarkovskii Theorem for $k=3$ is not a corollary to Theorem 2 if the alternate condition is used. That condition (5) may not be replaced by the assumption that $H$ and $K$ are mutually exclusive can be seen by the following. For the function $f:[0,1] \rightarrow[0,1]$, which is piecewise linear and contains the points $\left(0, \frac{1}{2}\right),\left(\frac{1}{2}, 1\right)$ and $(1,0)$, there do not exist mutually exclusive intervals $H$ and $K$ such that $f[H]$ contains $H \cup K$ and $f[K]$ contains $H$. To see this suppose $H$ and $K$ are such mutually exclusive intervals. By Theorem $2, K$ contains a periodic point of $f$ of period 3 . Note that $f^{3}$ has only four fixed points: $0, \frac{1}{2}, \frac{2}{3}$, and 1 . Since $\frac{2}{3}$ is a fixed point for $f, K$ must contain one of $0, \frac{1}{2}$, and 1 . We complete the proof by showing that each of these possibilities leads to a contradiction.

(1) Suppose 0 is in $K$. Then 1 is in $H$ since $f^{-1}(0)=\{1\}$ and $f[H]$ contains $K$. But since $f^{-1}(1)=\left\{\frac{1}{2}\right\}, \frac{1}{2}$ is in both $H$ and $K$.

(2) Suppose 1 is in $K$. Since $f^{-1}(1)=\left\{\frac{1}{2}\right\}, \frac{1}{2}$ is in $H$. Since $f^{-1}\left(\frac{1}{2}\right)=\left\{0, \frac{3}{4}\right\}$ and $H$ and $K$ do not intersect 0 is in $H$ and $\frac{3}{4}$ is in $K$. But, $f^{-1}(0)=\{1\}$ so 1 is in $H$.

(3) Suppose $\frac{1}{2}$ is in $K$. As before, one of 0 and $\frac{3}{4}$ is in $H$. Since $f^{-1}(0)=\{1\}$, if 0 is in $H$ then 1 is in both $H$ and $K$. Thus $\frac{3}{4}$ is in $H$. Then $f^{-1}\left(\frac{3}{4}\right)$ contains two points, $\frac{5}{8}$ and one less than $\frac{1}{2}$, so $P_{1}=\frac{5}{8}$ is in $H$. Since $f^{-1}\left(P_{1}\right)$ contains two points, $\frac{1}{8}$ and one between $\frac{5}{8}$ and $\frac{3}{4}, \frac{1}{8}$ is in $K$. Thus, $f^{-1}\left(\frac{1}{8}\right)=\frac{15}{16}$ is in $H$. Since $f^{-1}\left(\frac{15}{16}\right)$ contains two points, $\frac{17}{32}$ and one less than $\frac{1}{2}, P_{2}=\frac{17}{32}$ is in $H$. Continuing this process, we get a sequence $P_{1}, P_{2}, \ldots$ of points of $H$ which converges to $\frac{1}{2}$. Thus $\frac{1}{2}$ is in $H$.

4. Periodic points and indecomposability. In this section we show that under certain conditions the existence of a periodic point of period three in a mapping of a continuum $M$ to itself implies that $\lim \{M, f\}$ contains an indecomposable continuum. Of course the result is not true in general since a rotation of $S^{1}$ by 120 degrees yields a homeomorphism of $S^{1}$ and a copy of $S^{\mathbf{1}}$ for the inverse limit.

THEOREM 3. Suppose $f$ is a mapping of the continuum $M$ into itself and $x$ is a point of $M$ which is a periodic point of $f$ of period three. If $M$ is atriodic and hereditarily unicoherent, then $\lim \{M, f\}$ contains an indecomposable continuum. 
Moreover, the inverse limit is indecomposable if $\operatorname{cl}\left(\bigcup_{i>0} f^{i}\left[M_{1}\right]\right)=M$, where $M_{1}$ is the subcontinuum of $M$ irreducible from $x$ to $f(x)$.

PrOOF. Suppose $x$ is a periodic point of $f$ of period three. Denote by $M_{1}, M_{2}$ and $M_{3}$ subcontinua of $M$ irreducible from $x$ to $f(x), f(x)$ to $f^{2}(x)$ and $f^{2}(x)$ to $x$, respectively. Note that since $M$ is hereditarily unicoherent, $M_{1} \cap\left(M_{2} \cup M_{3}\right)=$ $\left(M_{1} \cap M_{2}\right) \cup\left(M_{1} \cap M_{3}\right)$ is a continuum, so there is a point $p$ common to all three continua.

The three continua $M_{1} \cap M_{2}, M_{2} \cap M_{3}$ and $M_{1} \cap M_{3}$ all contain the point $p$ so, since $M$ is atriodic, one of them is a subset of the union of the other two [15]. Suppose $M_{1} \cap M_{2}$ is a subset of $\left(M_{2} \cap M_{3}\right) \cup\left(M_{1} \cap M_{3}\right)=M_{3} \cap\left(M_{1} \cup M_{2}\right)=M_{3}$. (The last equality follows since $M_{3} \cap\left(M_{1} \cup M_{2}\right)$ is a subcontinuum of $M_{3}$ containing $x$ and $f^{2}(x)$ and $M_{3}$ is irreducible between $x$ and $\left.f^{2}(x)\right)$. Then, $M_{1} \cup M_{2}$ is a subset of $M_{3}$ for if not there is a point $t$ of $M_{1} \cup M_{2}$ such that $t$ is not in $M_{3}$. Since $M_{1} \cap M_{2}$ is a subset of $M_{3}, t$ is in $M_{1}$ or in $M_{2}$ but not in $M_{1} \cap M_{2}$. Suppose $t$ is in $M_{1}-\left(M_{1} \cap M_{2}\right)$. Since $t$ is not in $M_{3}, t$ is in $M_{1}-\left(M_{1} \cap M_{3}\right)$ and thus $t$ is in

$$
M_{1}-\left[\left(M_{1} \cap M_{2}\right) \cup\left(M_{1} \cap M_{3}\right)\right]=M_{1}-\left[M_{1} \cap\left(M_{2} \cup M_{3}\right)\right] .
$$

But, $M_{1} \cap\left(M_{2} \cup M_{3}\right)$ is a subcontinuum of $M_{1}$ containing $x$ and $f(x)$, so it contains $M_{1}$ since $M_{1}$ is irreducible between $x$ and $f(x)$. Thus, $M_{1}=M_{1} \cap\left(M_{2} \cup M_{3}\right)$ and so $M_{1} \cup M_{2}$ is a subset of $M_{3}$.

Note that $f\left[M_{1}\right]$ is a continuum containing $f(x)$ and $f^{2}(x)$, so $f\left[M_{1}\right] \cap M_{2}$ is a subcontinuum of $M_{2}$ containing these two points. Since $M_{2}$ is irreducible from $f(x)$ to $f^{2}(x), f\left[M_{1}\right] \cap M_{2}=M_{2}$. Therefore, $M_{2}$ is a subset of $f\left[M_{1}\right]$. Similarly, $f\left[M_{2}\right]$ contains $M_{3}$ and $f\left[M_{3}\right]$ contains $M_{1}$. However, since $M_{3}$ contains $M_{1} \cup M_{2}, M_{3}$ contains $x, f(x)$ and $f^{2}(x)$, so $f\left[M_{3}\right]$ contains $M_{1} \cup M_{2} \cup M_{3}$. Thus, $f^{n+2}\left[M_{1}\right]$ contains $f^{n+1}\left[M_{2}\right]$ which contains $f^{n}\left[M_{3}\right]$ which contains $M_{1} \cup M_{2} \cup M_{3}$ for $n=1,2,3, \ldots$ and so $\operatorname{cl}\left(\bigcup_{i>0} f^{i}\left[M_{1}\right]\right)=\operatorname{cl}\left(\bigcup_{i>0} f^{i}\left[M_{2}\right]\right)=\operatorname{cl}\left(\bigcup_{i>0} f^{i}\left[M_{3}\right]\right)$. Then, $H=\operatorname{cl}\left(\bigcup_{n>0} f^{n}\left[M_{1}\right]\right)$ is a continuum such that $f \mid H: H \rightarrow H$. Denote by $K$ the inverse limit, $\lim \{H, f \mid H\}$. We show that $K$ is indecomposable by showing the conditions of [6, Theorem 2, p. 267] are satisfied. Suppose $n$ is a positive integer and $e$ is a positive number. There is a positive integer $k$ such that if $t$ is in $H$ then $d\left(t, f^{k}\left[M_{3}\right]\right)<e$. Suppose $C$ is a subcontinuum of $H$ containing two of the three points, $x, f(x)$ and $f^{2}(x)$. Then $C$ contains one of $M_{1}, M_{2}$ and $M_{3}$. In any case $f^{2}[C]$ contains $M_{3}$, and thus, if $m=k+2, d\left(t, f^{m}[C]\right)<e$ for each $t$ in $H$. By Kuykendall's Theorem, $K$ is indecomposable.

THEOREM 4. If $f$ is a mapping of $[0,1]$ to $[0,1]$ and $f$ has a periodic point whose period is not a power of 2 , then $\lim \{([0,1], f)\}$ contains an indecomposable continuum. Moreover, for each positive integer $i$, there exists a mapping which has a periodic point of period $2^{i}$ and hereditarily decomposable inverse limit. ${ }^{1}$

PROOF. Suppose $f$ has a periodic point which has period $n$ and $n$ is not a power of 2 . Then, $n=2^{j}(2 k+1)$ for some $j, k \geq 0$, and $f^{2^{j}}$ has a periodic point of period $2 k+1$. By the Sarkovskii Theorem, $f^{2^{j}}$ has a periodic point of period 6 , so

\footnotetext{
${ }^{1}$ Added in proof: Theorem 4 first appeared, with a slightly different proof, as Theorem 1 of Chaos, periodicity, and snakelike continua by Marcy Barge and Joe Martin in a publication (MSRI 014-84) of the Mathematical Sciences Research Institute, Berkeley, California in January, 1984.
} 
$g=\left(f^{2^{j}}\right)^{2}$ has a periodic point of period 3. Since $\lim \{[0,1], f\}$ is homeomorphic to $\lim \{[0,1], g\}$, by Theorem $3 \lim \{[0,1], f\}$ contains an indecomposable continuum.

In the family of maps $f_{\mu}(x)=\mu x(1-x)$, for $2<\mu<\mu_{c} \sim 3.5699456 \ldots$ all the inverse limits for $\mu$ in this range are hereditarily decomposable and for each power of 2 , there is a map in this collection with a periodic point of period that power of 2. In fact for $2<\mu<3$ the inverse limit is an arc, for $3<\mu<\mu_{c}$ the inverse limit becomes, as $\mu$ increases, first a sinusoid, then a sinusoid to a double sinusoid, etc. For more details on this, see [9].

\section{REFERENCES}

1. James F. Davis, Atriodic acyclic continua and class W, Proc. Amer. Math. Soc. 90 (1984), 477-482.

2. James F. Davis and W. T. Ingram, An atriodic tree-like continuum with positive span which admits a monotone mapping to a chainable continuum, Fund. Math. (to appear).

3. Robert L. Devaney, An introduction to chaotic dynamical systems, Benjamin/Cummings, Menlo Park, Calif., 1986.

4. O. H. Hamilton, A fixed point theorem for pseudo arcs and certain other metric continua, Proc. Amer. Math. Soc. 2 (1951), 173-174.

5. W. T. Ingram, An atriodic tree-like continuum with positive span, Fund. Math. 77 (1972), 99-107.

6. Daniel P. Kuykendall, Irreducibility and indecomposability in inverse limits, Fund. Math. 84 (1973), 265-270.

7. Tien-Yien Li and James A. Yorke, Period three implies chaos, Amer. Math. Monthly 82 (1975), 985-992.

8. Wayne Lewis, Periodic homeomorphisms of chainable continua, Fund. Math. 117 (1983), 81-84.

9. Jack McBryde, Inverse limits on arcs using certain logistic maps as bonding maps, Master's Thesis, University of Houston, 1987.

10. Piotr Minc, A fixed point theorem for weakly chainable plane continua, preprint.

11. Sam B. Nadler, Examples of fixed point free maps from cells onto larger cells and spheres, Rocky Mountain J. Math. 11 (1981), 319-325.

12. David M. Read, Confluent and related mappings, Colloq. Math. 29 (1974), 233-239.

13. Helga Schirmer, A topologist's view of Sharkovsky's Theorem, Houston J. Math. 11 (1985), 385-395.

14. Michel Smith and Sam Young, Periodic homeomorphisms on T-like continua, Fund. Math. 104 (1979), 221-224.

15. R. H. Sorgenfrey, Concerning triodic continua, Amer. J. Math. 66 (1944), 439-460.

Department of Mathematics, University of Houston, Houston, Texas 77004 